\title{
The Effect of Anesthesia and Surgery on Postoperative Changes in Plasma biomarkers of neuronal injury, Alzheimer's disease, and inflammation in healthy subjects
}

\section{Wenguo Fan ( $\nabla$ fanweng@mail.sysu.edu.cn )}

Sun Yat-Sen University Guanghua School of Stomatology

\section{Lijia Mai}

Sun Yat-Sen University Guanghua School of Stomatology

Zhi Wu

Sun Yat-Sen University Guanghua School of Stomatology

Qiaomei Wu

Sun Yat-Sen University Guanghua School of Stomatology

\section{Xiaoping Yang}

Sun Yat-Sen University Guanghua School of Stomatology

\section{Wenzhen Gu}

Sun Yat-Sen University Guanghua School of Stomatology

Yifan HE

Sun Yat-Sen University Guanghua School of Stomatology

Xiao Zhu

Guangdong Medical College Zhanjiang Campus: Guangdong Medical University

\section{Guanghui Song}

The Affiliated Hospital of Qingdao University

\section{Fang Huang}

Sun Yat-Sen University Guanghua School of Stomatology

Hongwen $\mathrm{He}$

Sun Yat-Sen University Guanghua School of Stomatology

\section{Research}

Keywords: Anesthesia and Surgery, Neuronal injury, Neuroinflammation, Plasma biomarkers, Healthy subjects, PND

Posted Date: March 23rd, 2021 
DOI: https://doi.org/10.21203/rs.3.rs-317062/v1

License: (c) (1) This work is licensed under a Creative Commons Attribution 4.0 International License. Read Full License 


\section{Abstract}

Background: Anesthesia and surgery have been linked to neurological sequelae such as perioperative neurocognitive disorders (PND) and increased risk of Alzheimer's disease (AD). The exact mechanisms of PND remain ambiguous and controversial, which were deserved to explore further.

Methods: Healthy subjects undergoing general anesthesia for orthognathic surgery were prospectively randomized to receive propofol or sevoflurane for anesthetic maintenance. Blood samples were taken preoperatively and at 3,24 , and 48 hours after surgery. Neurofilament light (NFL), tau, Amyloid $\beta(A \beta) 40$, $A \beta 42$ and 21 inflammatory mediators in plasma were measured using highly sensitive assays.

Results: A total of 50 healthy subjects were enrolled. The mean (SD) age was $24.80(4.63)$ years. Plasma $\mathrm{NFL}$ increased at each measurement from a baseline mean (SD) of $22.3(20.4) \mathrm{pg} / \mathrm{mL}$ to a maximal mean (SD) level of 35.1 (28.7) pg/mL, a maximum increase of $599 \%$, at 3 hours postoperatively. NFL level began to decline at 24 hours, but remained higher at 48 hours. The levels of $A \beta 40$ and $A \beta 42$ decreased at 3 hours, and to minimum mean (SD) of $196.70(38.61) \mathrm{pg} / \mathrm{mL}$ and $8.01(1.66) \mathrm{pg} / \mathrm{mL}$ at 24 hours postoperatively, respectively. There were no significant differences in the concentrations of plasma tau after anesthesia and surgery. Plasma IL-6, IL-7, IL-8, IL-10, TNF-a, I-TAC and MIP-1 $\beta$ were significantly increased at 3 hours postoperatively and then declined, which had a similar trajectory with a return to baseline. The peak levels of NFL, IL-6, IL-8, TNF- $\alpha$ and MIP-1 $\beta$ correlated with duration of surgery. The peak plasma NFL level significantly correlated with the levels of IL-6 and IL-8.

Conclusions: In the healthy adults, general anesthesia and surgery were associated with an increase in $\mathrm{NFL}$, and a decrease in A 340 and $A \beta 42$ in the plasma. Elevated plasma NFL levels might be attributed to many of inflammatory mediators. The data indicate systemic inflammation after anesthesia and surgery may induce neuronal injury. These preliminary findings in healthy subjects could help us to understand the effects of anesthesia/surgery on brain and the potential mechanisms of PND.

Trial Registration: The study was registered in Chinese Clinical Trial Registry on Feb 11st, 2019 (ChiCTR1900021289).

\section{Background}

It has become a common belief that general anesthesia acts reversibly on the central nervous system, which does not cause neuronal damage. However, impairments in cognitive ability are common complications experienced after anesthesia and surgery, particularly in the elderly [1]. The faith in the safety of anesthesia and surgery on the brain has been at odds with the clinical observations. These postoperative neurological complications include any form of acute event (postoperative delirium) and cognitive decline diagnosed up to 30 days after the procedure (delayed neurocognitive recovery) and up to 12 months (postoperative neurocognitive disorder, POCD) [1, 2]. 
Now all forms of cognitive disorder above are referred to collectively as perioperative neurocognitive disorders (PND) [1]. The underlying pathogenesis of PND remains controversial. Growing evidence suggests that a systemic inflammatory reaction induced by surgical trauma may play important roles in PND [2-4]. In addition, data from animal and human studies have not been consistent in whether general anesthesia itself is a risk factor for PND [5-7]. There are other factors affect the prevalence of PND which include age, the depth of anesthesia, underlying disease, the types of surgery, and comorbidities [8].

Human biomarker studies hold promise for establishing causation and for risk stratification and monitoring pathogenic processes or outcome of treatment $[9,10]$. Neurofilament light chain (NFL) has been a promising fluid biomarker of brain damage in a wide variety of neurological disorders [11-14]. Amyloid $\beta(A \beta)$ and tau are involved in Alzheimer's disease (AD) pathology or neuronal injury, which have been used as biomarkers for identifying the earliest stages of the disease [15]. Hence, in the present study, we limited confounders known to affect the prevalence of PND, and enrolled healthy adult subjects undergoing anesthesia and surgery. Using highly sensitive assays to measure NF-L, $A \beta$, tau and 21 inflammatory mediators in blood, the goals of our study were to (1) examine and compare levels of plasma biomarkers of neuronal injury, $A D$ and inflammation, (2) examine the potential relationships between the markers of neuronal injury and inflammation.

\section{Methods}

\section{Study participants}

The study was approved by Ethics Committee of Hospital of Stomatology, Sun Yet-sen University, and registered with http://www.chictr.org.cn/index.aspx (ChiCTR1900021289). Fifty adult subjects with the American Society of Anesthesiologists (ASA) status I were enrolled who were undergoing orthognathic surgery.

All participants provided written informed consent, and underwent an evaluation that consisted of medical history, physical and neurological examinations, laboratory tests and neuropsychological assessments. Inclusion and exclusion criteria are provided in Additional file 1. The participants were assigned to receive general anesthesia that consisted of either a volatile agent (sevoflurane) or intravenous anesthesia (propofol). The general anesthesia type was chosen according to the preference of the anesthesiologist. For orthognathic surgery, general anesthesia was usually administered in combination with local nerve blocks (Articaine).

Blood samples were taken consecutively before surgical anesthesia (baseline), after anesthesia induction (0 hour), at 3, 24, and 48 hours after surgery, and stored on ice in vacutainer tubes (Becton Dickinson) containing the anticoagulant ethylenediaminetetraacetic acid (EDTA). Within 1 hour, these tubes were centrifuged at $4^{\circ} \mathrm{C}$ for 10 minutes at 3000 revolutions per minute, and then aliquoted and stored at $-80^{\circ} \mathrm{C}$ for subsequent batch analysis.

\section{Inflammatory mediator Measurement}


The plasma was analyzed for inflammatory mediator levels with Luminex xMAP technology (Luminex Corp). Commercial MILLIPLEX MAP kits (Millipore; Billerica, MA, USA) were used in this study. We assessed a broad spectrum of inflammatory markers including Fractalkine, GM-CSF, IFN- - , IL-1 $\beta$, IL-2, IL-4, IL-5, IL-6, IL-7, IL-8/CXCL8, IL-10, IL-12 (p70), IL-13, IL-17A, IL-21, IL-23, I-TAC, MIP-1a, MIP-1 $\beta$, MIP-3a, TNFa (Cat. No. HSTCMAG28SPMX21). The multiplexed assays were performed according to the manufacturer's instructions. All plasma samples were analyzed using a LiquiChip Luminex $200^{\text {tm }}$ Workstation (Qiagen,Valencia, CA, USA).

\section{Neurology Assays}

Plasma NFL, t-tau, $A \beta 40$ and $A \beta 42$ levels were measured on the Quanterix Simoa-HD1 Platform (Quanterix, MA, USA) at GBIO (Hangzhou, China). The multiplex assays Neurology 3-Plex A Kits (Cat No.101995) and NFL Kit (Cat No.102258) were purchased from Quanterix and used according to the manufacture's instruction. Samples were diluted at 1:4 ratio and were performed on single run using 1 batch of reagents. All plasma analyses were performed by skilled laboratory technicians blinded to clinical information.

\section{Statistical analysis}

Data were presented as mean ( \pm standard deviation, SD). A repeated measure one-way analysis of variance (ANOVA) was applied to statistically compare longitudinal changes after surgery. The Bonferroni post-hoc test was used to test statistical differences only when the overall test was significant. Correlations between variables were performed by Pearson correlation coefficient. A P value of less than 0.05 was taken to indicate significance. Tests were performed using SPSS 20.0 version (IBM Corporation, Chicago, IL, USA).

\section{Results}

\section{Demographics}

A total of 71 subjects were screened. There were 50 participants enrolled in this study. Enrollment is summarized in a summary and flow diagram in Additional file 2 (Figure S). There were 24 males and 26 females; The mean age of the subjects was 24.80 (4.63) years. Perioperative plasma samples were available for a total of 50 consecutive participants. Baseline and intraoperative characteristics of the study subjects were summarized in Table 1. Patients were euthermic throughout, and none experienced unusual changes in physiology. Procedures were without complications from either the anesthesia or surgery.

\section{Anesthesia and surgery induce neurological damage}

The mean plasma NF-L, t-tau, A $\beta 40$ and $A \beta 42$ concentrations were summarized in Table 2. Plasma NF-L showed a significant increase at each measurement from a baseline mean (SD) of 3.73 (1.7) $\mathrm{pg} / \mathrm{mL}$ to a maximal mean (SD) of $22.74(18.55) \mathrm{pg} / \mathrm{mL}(\mathrm{P}<0.001)$ at 3 hours postoperatively. (Table 2). Mean 
changes from baseline were $599 \%$ at 3 hours; $562 \%$ at 24 hours; and $540 \%$ at 48 hours postoperatively. NF-L levels began to decline after 24 hours, but remained higher levels after 48 hours. (Figure 1).

The concentrations of plasma tau were statistically unchanged after anesthesia and surgery (Table 2). The baseline mean (SD) was 3.71(1.06) pg/mL and a maximal mean (SD) was 4.67(6.61) $\mathrm{pg} / \mathrm{mL}(\mathrm{p}=0.35)$ at 24 hours postoperatively. Mean tau concentrations fluctuated by less than $30 \%$.

Mean plasma $A \beta 40$ and $A \beta 42$ levels was decreased at 3 hours, and at 24 hours postoperatively to minimum mean (SD) of 196.70 (38.61) and 8.01(1.66), respectively. (Table 2). $A \beta 40$ and $A \beta 42$ of mean changes from baseline were $-22 \%$ and -30 at 24 hours, $-15.5 \%$ and $-21.6 \%$ at 48 hours after surgery, respectively. (Figure 1 ).

\section{Anesthesia and surgery trigger systemic inflammatory response}

Plasma IL-6, IL-7, IL-8, IL-10, TNF-a, I-TAC and MIP-1 $\beta$ were significantly increased at 3 hours postoperatively (Table3, Figure 3 ) and then declined, which had a similar trajectory with a return to baseline, only the levels of I-TAC and MIP-1 $\beta$ returned below the baseline at 48 hours after surgery. The changes from baseline were maximal at 3 hours, increases of $697.3 \%, 47.3 \%, 189.9 \%, 217.7 \%, 44.5 \%$ $6.6 \%$, and $58.6 \%$, respectively. Plasma levels of Fractalkine, GM-CSF, IFN- $y$, IL-2, IL-4, IL-5, IL-12, IL-13, IL17A, IL-21, IL-23, MIP-3a/CCL20 were not significantly changed over time after anesthesia and surgery (Table S囚provided in Additional file 3). Plasma IL-1 $\beta$ and MIP-1 a concentrations were below the detection threshold for most samples at baseline and during the postoperative period.

\section{Neuronal damage might be influenced by duration of surgery/anesthesia}

Duration of surgery varied between 1.08 and 5.50 hour. The peak levels of NFL, IL-6, IL-8, TNF- $a$ and MIP$1 \beta$ correlated with duration of surgery $(r=0.64, p<0.001 ; r=0.65, P<0.001 ; r=0.73, P<0.001 ; r=0.57$, $P<0.001 ; r=0.57, P<0.001$; Figure 3).

There was no correlation between the peak levels of $A \beta 40, A \beta 42, I L 7, I-T A C, I L-10$ and surgery duration postoperatively $(r=0.24 P=0.1 ; r=0.07, P=0.63 ; r=0.087, P=0.55 ; r=0.066, P=0.65 ; r=0.24, P=0.10$, respectively, Figure 4).

\section{Neuronal injury might be largely driven by IL-6 and IL-8}

The peak plasma NFL level significantly correlated with the levels of IL- 6 and IL-8 $(r=0.34, P<0.017 ; r$ $=0.32, P<0.02$; Figure 5). There was no correlation between the peak plasma NFL level and IL-7, IL-10, TNF- $\alpha$ and MIP-1 $\beta$ and I-TAC ( $r=0.048, P=0.74 ; r=0.13, P=0.39 ; r=0.21, P=0.15 ; r=-0.121, P=0.41 ;$ Figure 5).

\section{Discussion}

In the present study, we limited confounders such as the ages, underlying disease, the type of surgery and comorbidities known to affect the prevalence of PND, and enrolled healthy adult subjects undergoing 
anesthesia and surgery. The present results demonstrated that plasma NFL was significantly increased, but $A \beta 42$ and $A \beta 40$ were decreased after anesthesia and surgery. The changes of biomarkers of neuronal injury might be attributed to the changes of multiple inflammatory mediators after surgery.

NFL is the major constituents of the neuronal cytoskeleton [16], which is detected in the cerebrospinal fluid (CSF) and that increased CSF NFL levels are associated with neuronal injury in some neurodegenerative disorders, such as multiple sclerosis, HIV infection, and Alzheimer disease [17]. Plasma NFL levels are associated with the severity of injured and/or degenerating neurons and correlated highly with CSF levels $[18,19]$. In the present study, we found the NFL levels in plasma increased rapidly in response to anesthesia and surgery. In general, our results of NFL were in agreement with those reported by Evered et al [20], who measured in elderly surgical patients. In addition, our results confirmed a strong positive correlation between NFL levels and duration of anesthesia/surgery. Since the measurement of serum NFL may be useful to assess the severity of neuronal injury following traumatic brain injury [21] and ischemic stroke [22], PND is believed to be multifactorial and involves age and healthy state, and NFL is sensitive for neuro injury, our results from healthy adult subjects suggest that anesthesia and surgery are responsible for the neurotoxicity, although DiMeglio's study showed that serum NFL concentration did not change significantly after cardiac surgery [23].

Neuritic plaques containing $A \beta$ and neurofibrillary tangles consisting of tau protein are the primary neuropathologic criteria for $A D$ diagnosis [24]. Both $A \beta 42$ and $A \beta 40$ isoforms in the CSF and/or blood have been used as biomarkers for the identification of the earliest stages of the certain forms of AD $[9$, 25-27]. Evered's study showed that plasma levels of $A \beta 42$ and $A \beta 40$ were significantly lower in patients with POCD at 3 months than those without POCD after cardiac surgery [28]. The lower plasma levels of $A \beta$ may indicate premorbid neurodegenerative disease as $A \beta$ deposited selectively in the brain [29]. In the present study, the levels of $A \beta 42$ and $A \beta 40$ decreased over time after surgery, which remain lower levels at 48 hours postoperatively. There are studies showing that the level of plasma $A \beta$ is associated with age $[15,30]$. Moreover, the first signs of $A D$ pathology and cognitive decline may occur from around 50-60 years of age [31,32]. The decreased blood $A \beta$ of Evered's results may be involved in age, since the mean age of the patients enrolled was 68.0 years [28]. Our findings showed lower level of plasma $A \beta$ obtained from young healthy adults, which are unlikely attributed to $A \beta$ accumulation. A recent study showed that exposure to surgery with general anesthesia during adult life did not induce increased $A \beta$ deposition in brain [33]. In addition, Pikwer's study showed that there were no significant effects on A $\beta$ in CSF after surgery and anesthesia (5 hours after induction) [34]. These data suggest that surgery and anesthesia may be involved in the complex mechanisms of $A \beta$ metabolism, which remain to be explored.

Tau protein is primarily localized in CNS neurons and contributes to axonal integrity, which is considered as an important biomarker for neurodegenerative disease and brain injury [35]. Preclinical and clinical studies suggest that anesthesia and/or surgery have effect on this biomarker of $A D$, the changes of which might be associated with PND [36]. Previous studies have showed that blood tau increased rapidly from baseline postoperatively [20], and even remained elevated at 7 days and three months [23] in the 
elderly. In the present study, we did not detect a difference in levels of tau over time. A similar finding has been reported by Pikwer et al. [34]. They demonstrated that anesthesia and surgery have no effects on tau in adults. These results suggest that tau metabolism may be related to age in that aging brains are vulnerable to anesthesia and surgery.

Surgery and anesthesia can trigger a systemic inflammatory response which is coordinated by the immune system and mediated by endogenous mediators such as inflammatory cytokines [37]. For example, Hirsch's study showed that statistically significant changes compared to baseline were present in IL-5, IL-6, IL-8, IL-10, monocyte chemotactic protein (MCP)-1, MIP-1a in plasma of patients undergoing major knee surgery who received spinal anesthesia with intravenous sedation (propofol) [38]. There are other studies showing that the serum levels of cytokines, such as IL-1ß, IL-2, IL- 6 and IL-8, increased after anesthesia and surgery [39-42]. In the present study, we observed dynamic changes over time after anesthesia and surgery in specific plasma inflammatory biomarkers, including IL-6, IL-7, IL-8, IL-10, TNF-a, I-TAC (CXCL11) and MIP-1 $\beta$. The inflammatory mediators were increased and peaked at 3 hours, and many of the cytokines were not restored baseline at 48 hours postoperatively. IL-10 trajectory matched other proinflammatory biomarkers in plasma, which is an anti-inflammatory cytokine that maintains the balance of the immune response [43]. The data suggests a substantial activation of key pathways of the immune response following surgery and anesthesia. Moreover, we further observed that there were correlations between plasma inflammatory mediators (IL-6, IL-7, IL-8, IL-10) and duration of surgery and anesthesia. These results suggest that plasma levels of IL-6, IL-7, IL-8, IL-10 may be useful markers of the magnitude of surgical stress response to trauma and injury. In general, our results were consistent with previous studies, although there were contradictory results obtained in the pattern and extent of inflammatory response [44], which may be due to different types of surgery, underlying diseases, research methods and age of subjects.

Neuroinflammation has become a key hallmark of neurological complications including PND [4]. Surgery is known to induce a systemic inflammatory response causing blood-brain barrier breakdown and then triggering neuroinflammation $[38,45,46]$. In the present study, we demonstrated that there was a positive correlation between plasma concentrations of NFL and inflammatory cytokines (IL-6 and IL-8). The changes of biomarkers of neuronal injury might be attributed to increase of inflammatory cytokines IL-6 and IL-8. These findings lead to possibility that systemic inflammation might have profound impact on the brain after anesthesia and surgery. Experimental and clinical studies have suggested that different anesthetics may modulate immune signaling pathways, which can directly cause immune suppression by influencing the functions of immunocompetent cells and inflammatory mediator gene expression and secretion $[47,48]$. Volatile anesthetics have been thought to have antiinflammatory properties $[49,50]$. In addition, a latest research showed that general anesthesia (sevoflurane) without surgery in healthy volunteers did not provoke an inflammatory state or neuronal injury in the early hours after exposure [10]. Another study in healthy subjects without any surgical intervention or other nociceptive stimuli demonstrated that propofol exerted a partly pro-inflammatory but also slightly anti-inflammatory effect on the immune system [51]. Since anesthetics may have anti- 
inflammatory effects on the immune system, our results obtained in healthy subjects suggest that systemic inflammation induced by operative trauma might be main culprit causing neuronal injury.

\section{Limitations}

Although anesthesia and surgery have been proposed to increase the incidence of PND [7, 52], we did not observe a corresponding behavioral phenotype to the changes we measured in a short period. Whether the changes have a prolonged effect on brain and cause cognitive dysfunction remained to be evaluated. Because of anesthesia and the accompanying surgical interventions, we cannot differentiate any adverse effects of the surgical trauma from those specifically associated with the use of anesthetic agents. Moreover, due to invasiveness and low availability of the testing CSF biomarkers, the biomarkers of systemic inflammation might not be able to evaluate neuroinflammation, although systemic inflammatory processes have been linked to brain homeostasis and brain injury [53]. The causal relationship between the changes of plasma cytokines and neuronal damage will be required to determine. Clinical studies designed properly could elucidate the effects of inflammation on the pathogenesis of PND. The use of animals in research is also essential to further elucidate the underlying cellular mechanisms.

\section{Conclusions}

In summary, we limited some confounders which might affect the prevalence of PND in the present study. This is the first study to report that a neuronal injury has taken place after anesthesia and surgery in the healthy subjects, although the neurotoxicity might sustain in a short term. Moreover, the neuronal damage might be largely driven by many of cytokines levels, which was influenced by duration of anesthesia and extent of surgical trauma. The data provide highly valuable information in an understanding of neurological damage by anesthesia/surgery and the potential mechanisms of PND.

\section{Abbreviations}

$A \beta$ : Amyloid $\beta$; AD: Alzheimer's disease; ASA: American Society of Anesthesiologists; BMl: Body mass index; CSF: cerebrospinal fluid; granulocyte-macrophage colony-stimulating factor (GM-CSF); I-TAC: IFNinducible T cellachemoattractant; IL: Interleukine; MIP: macrophage inflammatory protein; NFL: Neurofilament light chain; PND: perioperative neurocognitive disorders; POCD: postoperative neurocognitive disorder; TNF-a: tumor necrosis factor a

\section{Declarations}

\section{Acknowledgments}

We thank all the study participants, as well as the clinical staff, for making the study possible.

\section{Authors' contributions}


WF developed and designed the study and wrote the manuscript. WF, LM and ZW had full access to all of the data in the study and take responsibility for the integrity of the data and the accuracy of the data analysis. WF, LM and ZW contributed equally to this work. WF and ZW performed analyses. $\mathrm{XZ}$ and FH prepared the datasets. GS and $\mathrm{YH}$ performed necessary experiments. $\mathrm{HH}$ and $\mathrm{XY}$ provided supervision and direction for the whole study. All authors read and approved the final manuscript.

\section{Funding}

This work was supported partly by the National Natural Science Foundation of China (No. 81771098 and 81541153).

\section{Availability of data and materials}

The data supporting the conclusions of this article are included within the article and additional file.

\section{Ethics approval and consent to participate}

This study was approved by Ethics Committee of Hospital of Stomatology, Sun Yet-sen University (GHKQ201812-K2-01) and we followed all appropriate protocols.

\section{Consent for publication}

All the co-authors and participants have given their consent for publication.

\section{Competing interests}

The authors declare that they have no conflict of interest.

\section{References}

1. Evered L, Silbert B, Knopman DS, et al. Recommendations for the Nomenclature of Cognitive Change Associated with Anaesthesia and Surgery-2018. Anesthesiology. 2018;129(5):872-879.

2. Safavynia SA, Goldstein PA. The Role of Neuroinflammation in Postoperative Cognitive Dysfunction: Moving From Hypothesis to Treatment. Front Psychiatry. 2018;9:752.

3. Forsberg A, Cervenka S, Jonsson Fagerlund $M$, et al. The immune response of the human brain to abdominal surgery. Annals of neurology. 2017;81(4):572-582.

4. Subramaniyan S, Terrando N. Neuroinflammation and Perioperative Neurocognitive Disorders. Anesthesia and analgesia. 2019;128(4):781-788.

5. Ologunde R, Ma D. Do inhalational anesthetics cause cognitive dysfunction? Acta anaesthesiologica Taiwanica : official journal of the Taiwan Society of Anesthesiologists. 2011;49(4):149-153.

6. Zhang J, Tan H, Jiang W, Zuo Z. The choice of general anesthetics may not affect neuroinflammation and impairment of learning and memory after surgery in elderly rats. Journal of 
neuroimmune pharmacology : the official journal of the Society on Neurolmmune Pharmacology. 2015;10(1):179-189.

7. Belrose JC, Noppens RR. Anesthesiology and cognitive impairment: a narrative review of current clinical literature. BMC anesthesiology. 2019;19(1):241.

8. Hudetz JA, Patterson KM, Amole O, Riley AV, Pagel PS. Postoperative cognitive dysfunction after noncardiac surgery: effects of metabolic syndrome. Journal of anesthesia. 2011;25(3):337-344.

9. Shaw LM, Korecka M, Clark CM, Lee VM, Trojanowski JQ. Biomarkers of neurodegeneration for diagnosis and monitoring therapeutics. Nat Rev Drug Discov. 2007;6(4):295-303.

10. Deiner S, Baxter MG, Mincer JS, et al. Human plasma biomarker responses to inhalational general anaesthesia without surgery. British journal of anaesthesia. 2020.

11. Preische O, Schultz SA, Apel A, et al. Serum neurofilament dynamics predicts neurodegeneration and clinical progression in presymptomatic Alzheimer's disease. Nature medicine. 2019;25(2):277-283.

12. Zetterberg H. Neurofilament Light: A Dynamic Cross-Disease Fluid Biomarker for Neurodegeneration. Neuron. 2016;91(1):1-3.

13. Zerr I, Schmitz M, Karch A, et al. Cerebrospinal fluid neurofilament light levels in neurodegenerative dementia: Evaluation of diagnostic accuracy in the differential diagnosis of prion diseases. Alzheimer's \& dementia : the journal of the Alzheimer's Association. 2018;14(6):751-763.

14. Bacioglu M, Maia LF, Preische O, et al. Neurofilament Light Chain in Blood and CSF as Marker of Disease Progression in Mouse Models and in Neurodegenerative Diseases. Neuron. 2016;91(2):494496.

15. Lue LF, Pai MC, Chen TF, et al. Age-Dependent Relationship Between Plasma Abeta40 and Abeta42 and Total Tau Levels in Cognitively Normal Subjects. Frontiers in aging neuroscience. 2019;11:222.

16. Liu Q, Xie F, Siedlak SL, et al. Neurofilament proteins in neurodegenerative diseases. Cellular and molecular life sciences : CMLS. 2004;61(24):3057-3075.

17. Bridel $\mathrm{C}$, van Wieringen WN, Zetterberg $\mathrm{H}$, et al. Diagnostic Value of Cerebrospinal Fluid Neurofilament Light Protein in Neurology: A Systematic Review and Meta-analysis. JAMA neurology. 2019.

18. Rojas JC, Karydas A, Bang J, et al. Plasma neurofilament light chain predicts progression in progressive supranuclear palsy. Annals of clinical and translational neurology. 2016;3(3):216-225.

19. PiehI F, Kockum I, Khademi M, et al. Plasma neurofilament light chain levels in patients with MS switching from injectable therapies to fingolimod. Mult Scler. 2018;24(8):1046-1054.

20. Evered L, Silbert B, Scott DA, Zetterberg H, Blennow K. Association of Changes in Plasma Neurofilament Light and Tau Levels With Anesthesia and Surgery: Results From the CAPACITY and ARCADIAN Studies. JAMA neurology. 2018;75(5):542-547.

21. Shahim P, Gren M, Liman V, et al. Serum neurofilament light protein predicts clinical outcome in traumatic brain injury. Scientific reports. 2016;6:36791. 
22. Tiedt S, Duering M, Barro $C$, et al. Serum neurofilament light: A biomarker of neuroaxonal injury after ischemic stroke. Neurology. 2018;91(14):e1338-e1347.

23. DiMeglio M, Furey W, Hajj J, et al. Observational study of long-term persistent elevation of neurodegeneration markers after cardiac surgery. Scientific reports. 2019;9(1):7177.

24. Long JM, Holtzman DM. Alzheimer Disease: An Update on Pathobiology and Treatment Strategies. Cell. 2019;179(2):312-339.

25. Janelidze S, Zetterberg $\mathrm{H}$, Mattsson N, et al. CSF Abeta42/Abeta40 and Abeta42/Abeta38 ratios: better diagnostic markers of Alzheimer disease. Annals of clinical and translational neurology. 2016;3(3):154-165.

26. Olsson B, Lautner R, Andreasson U, et al. CSF and blood biomarkers for the diagnosis of Alzheimer's disease: a systematic review and meta-analysis. The Lancet Neurology. 2016;15(7):673-684.

27. Palmqvist S, Janelidze S, Stomrud E, et al. Performance of Fully Automated Plasma Assays as Screening Tests for Alzheimer Disease-Related beta-Amyloid Status. JAMA neurology. 2019.

28. Evered LA, Silbert BS, Scott DA, et al. Plasma amyloid beta42 and amyloid beta40 levels are associated with early cognitive dysfunction after cardiac surgery. The Annals of thoracic surgery. 2009;88(5):1426-1432.

29. Kawarabayashi T, Younkin LH, Saido TC, Shoji M, Ashe KH, Younkin SG. Age-dependent changes in brain, CSF, and plasma amyloid (beta) protein in the Tg2576 transgenic mouse model of Alzheimer's disease. The Journal of neuroscience : the official journal of the Society for Neuroscience. 2001;21(2):372-381.

30. Mayeux R, Honig LS, Tang MX, et al. Plasma A[beta]40 and A[beta]42 and Alzheimer's disease: relation to age, mortality, and risk. Neurology. 2003;61(9):1185-1190.

31. Jansen WJ, Ossenkoppele R, Knol DL, et al. Prevalence of cerebral amyloid pathology in persons without dementia: a meta-analysis. Jama. 2015;313(19):1924-1938.

32. Caselli RJ, Dueck AC, Osborne D, et al. Longitudinal modeling of age-related memory decline and the APOE epsilon4 effect. The New England journal of medicine. 2009;361(3):255-263.

33. Sprung J, Warner DO, Knopman DS, et al. Exposure to surgery with general anaesthesia during adult life is not associated with increased brain amyloid deposition in older adults. British journal of anaesthesia. 2020;124(5):594-602.

34. Pikwer A, Castegren M, Namdar S, Blennow K, Zetterberg H, Mattsson N. Effects of surgery and propofol-remifentanil total intravenous anesthesia on cerebrospinal fluid biomarkers of inflammation, Alzheimer's disease, and neuronal injury in humans: a cohort study. Journal of neuroinflammation. 2017;14(1):193.

35. Randall J, Mortberg E, Provuncher GK, et al. Tau proteins in serum predict neurological outcome after hypoxic brain injury from cardiac arrest: results of a pilot study. Resuscitation. 2013;84(3):351-356.

36. Evered L, Silbert B, Scott DA, Ames D, Maruff P, Blennow K. Cerebrospinal Fluid Biomarker for Alzheimer Disease Predicts Postoperative Cognitive Dysfunction. Anesthesiology. 2016;124(2):353361. 
37. Lin E, Calvano SE, Lowry SF. Inflammatory cytokines and cell response in surgery. Surgery. 2000;127(2):117-126.

38. Hirsch J, Vacas S, Terrando N, et al. Perioperative cerebrospinal fluid and plasma inflammatory markers after orthopedic surgery. Journal of neuroinflammation. 2016;13(1):211.

39. Zura M, Kozmar A, Sakic K, Malenica B, Hrgovic Z. Effect of spinal and general anesthesia on serum concentration of pro-inflammatory and anti-inflammatory cytokines. Immunobiology. 2012;217(6):622-627.

40. Danielson M, Reinsfelt B, Westerlind A, Zetterberg H, Blennow K, Ricksten SE. Effects of methylprednisolone on blood-brain barrier and cerebral inflammation in cardiac surgery-a randomized trial. Journal of neuroinflammation. 2018;15(1):283.

41. Hudetz JA, Gandhi SD, Iqbal Z, Patterson KM, Pagel PS. Elevated postoperative inflammatory biomarkers are associated with short- and medium-term cognitive dysfunction after coronary artery surgery. Journal of anesthesia. 2011;25(1):1-9.

42. Ji MH, Yuan HM, Zhang GF, et al. Changes in plasma and cerebrospinal fluid biomarkers in aged patients with early postoperative cognitive dysfunction following total hip-replacement surgery. Journal of anesthesia. 2013;27(2):236-242.

43. O'Garra A, Barrat FJ, Castro AG, Vicari A, Hawrylowicz C. Strategies for use of IL-10 or its antagonists in human disease. Immunol Rev. 2008;223:114-131.

44. Liu X, Yu Y, Zhu S. Inflammatory markers in postoperative delirium (POD) and cognitive dysfunction (POCD): A meta-analysis of observational studies. PloS one. 2018;13(4):e0195659.

45. Terrando N, Eriksson LI, Ryu JK, et al. Resolving postoperative neuroinflammation and cognitive decline. Annals of neurology. 2011;70(6):986-995.

46. Alam A, Hana Z, Jin Z, Suen KC, Ma D. Surgery, neuroinflammation and cognitive impairment. EBioMedicine. 2018;37:547-556.

47. Yuki K, Eckenhoff RG. Mechanisms of the Immunological Effects of Volatile Anesthetics: A Review. Anesthesia and analgesia. 2016;123(2):326-335.

48. Stollings LM, Jia LJ, Tang P, Dou H, Lu B, Xu Y. Immune Modulation by Volatile Anesthetics. Anesthesiology. 2016;125(2):399-411.

49. O'Gara B, Talmor D. Lung protective properties of the volatile anesthetics. Intensive care medicine. 2016;42(9):1487-1489.

50. Strosing KM, Faller S, Gyllenram V, et al. Inhaled Anesthetics Exert Different Protective Properties in a Mouse Model of Ventilator-Induced Lung Injury. Anesthesia and analgesia. 2016;123(1):143-151.

51. Kallioinen M, Scheinin A, Maksimow M, et al. The influence of dexmedetomidine and propofol on circulating cytokine levels in healthy subjects. BMC anesthesiology. 2019;19(1):222.

52. Mashour GA, Woodrum DT, Avidan MS. Neurological complications of surgery and anaesthesia. British journal of anaesthesia. 2015;114(2):194-203. 
53. Pluvinage JV, Wyss-Coray T. Systemic factors as mediators of brain homeostasis, ageing and neurodegeneration. Nat Rev Neurosci. 2020;21(2):93-102.

\section{Tables}

Table 1. Clinical characteristics of the study sample

\begin{tabular}{|ll|}
\hline Subjects Characteristics (N=50) & \\
\hline Age, mean (SD), years & $24.80(4.63)$ \\
\hline Sex-male/female no. & $24 / 26$ \\
\hline Body Weight, mean (SD), kg & $57.26(9.83)$ \\
\hline Body mass index, mean (SD), kg/m² & $20.60(1.97)$ \\
\hline Duration of surgery, mean (SD), hour & $2.50(0.91)$ \\
\hline General anesthesia type: propofol /sevoflurane & $23 / 27$ \\
\hline maxillary osteotomy no.(\% of total) & $1(2 \%)$ \\
\hline mandibular osteotomy no. (\% of total) & $22(44 \%)$ \\
\hline Combined maxillary mandibular osteotomies no. (\% of total) & $27(54 \%)$ \\
\hline
\end{tabular}

Table 2. Plasma Levels of the biomarkers of neuronal injury and AD perioperatively.

\begin{tabular}{|c|c|c|c|c|c|c|}
\hline Biomarker & baseline & 0 & $3 \mathrm{~h}$ & $24 \mathrm{~h}$ & $48 \mathrm{~h}$ & $\begin{array}{l}P \text { value } \\
(F)\end{array}$ \\
\hline NF-L & $\begin{array}{l}3.73 \\
(1.70)\end{array}$ & $\begin{array}{l}3.20 \\
(1.51)\end{array}$ & $\begin{array}{l}22.74^{\star \star} \\
(18.55)\end{array}$ & $\begin{array}{l}21.72 \star \star \star \\
(11.74)\end{array}$ & $\begin{array}{l}20.63^{\star *} \\
(11.06)\end{array}$ & $\begin{array}{l}₫ 0.001 \\
(39.102)\end{array}$ \\
\hline t-tau & $\begin{array}{l}3.71 \\
(1.06)\end{array}$ & $\begin{array}{l}3.56 \\
(1.51)\end{array}$ & $\begin{array}{l}4.44 \\
(1.65)\end{array}$ & $\begin{array}{l}4.67 \\
(6.61)\end{array}$ & $\begin{array}{l}3.81 \\
(1.14)\end{array}$ & $\begin{array}{l}0.35 \\
(1.113)\end{array}$ \\
\hline$A \beta 40$ & $\begin{array}{l}257.82 \\
(46.90)\end{array}$ & $\begin{array}{l}233.70 \\
(51.96)\end{array}$ & $\begin{array}{l}241.55 \\
(41.23)\end{array}$ & $\begin{array}{l}196.70 * \star \\
(38.61)\end{array}$ & $\begin{array}{l}212.88^{\star \star} \\
(37.92)\end{array}$ & $\begin{array}{l}₫ 0.001 \\
(14.805)\end{array}$ \\
\hline$A \beta 42$ & $\begin{array}{l}11.72 \\
(2.07)\end{array}$ & $\begin{array}{l}10.75^{\star} \\
(2.34)\end{array}$ & $\begin{array}{l}11.24 \\
(2.02)\end{array}$ & $\begin{array}{l}8.01^{* \star} \\
(1.66)\end{array}$ & $\begin{array}{l}9.03^{* *} \\
(1.56)\end{array}$ & $\begin{array}{l}\otimes 0.001 \\
(31.572)\end{array}$ \\
\hline
\end{tabular}

Values are presented as arithmetic mean $( \pm S D)$ concentrations $(\mathrm{pg} / \mathrm{mL})$ 
Compare to baseline ${ }^{\star} \mathrm{P}<0.05,{ }^{\star} * \mathrm{P}<0.01$.

Table 3. Plasma levels of inflammatory biomarkers over time

\begin{tabular}{|c|c|c|c|c|c|c|}
\hline Biomarker & baseline & Oh & $3 \mathrm{~h}$ & $24 \mathrm{~h}$ & $48 \mathrm{~h}$ & $\begin{array}{l}\text { P Value } \\
\text { (F) }\end{array}$ \\
\hline \multirow[t]{2}{*}{ IL-6 } & 2.42 & 2.54 & $14.58^{\star \star}$ & 5.26 & 3.47 & $\varangle 0.001$ \\
\hline & $(1.52)$ & $(1.70)$ & $(25.54)$ & (3.72) & $(2.13)$ & $(9.328)$ \\
\hline \multirow[t]{2}{*}{ IL-7 } & 9.93 & $19.21^{\star \star}$ & $13.02^{*}(5.27)$ & 11.00 & 10.31 & $\otimes 0.001$ \\
\hline & $(4.98)$ & $(11.26)$ & & (5.13) & $(5.24)$ & $(15.120)$ \\
\hline \multirow[t]{2}{*}{ IL-8 } & 3.11 & 3.24 & $8.57^{\star \star}$ & 3.93 & 3.52 & $\bowtie 0.001$ \\
\hline & $(0.97)$ & $(1.19)$ & $(6.58)$ & $(1.43)$ & $(1.00)$ & $(26.559)$ \\
\hline \multirow[t]{2}{*}{ IL-10 } & 9.51 & 9.83 & $21.64^{\star \star}(36.06)$ & 13.75 & 10.82 & 0.003 \\
\hline & $(4.91)$ & $(4.72)$ & & $(8.87)$ & $(6.12)$ & $(4.200)$ \\
\hline \multirow[t]{2}{*}{ TNF-a } & 4.66 & 4.84 & $6.57^{\star \star}$ & 4.13 & 4.12 & 0.002 \\
\hline & $(1.26)$ & $(1.35)$ & (7.10) & $(1.09)$ & $(1.13)$ & $(4.332)$ \\
\hline \multirow[t]{2}{*}{ I-TAC } & 23.42 & 24.59 & $24.27(9.96)$ & 20.17 & $17.06^{\star \star}(4.84)$ & $\varangle 0.001$ \\
\hline & $(8.87)$ & $(9.21)$ & & (7.13) & & $(7.514)$ \\
\hline \multirow[t]{2}{*}{ MIP-1 $\beta$} & 30.88 & 31.81 & $48.68^{* \star}$ & 29.21 & 28.79 & 0.015 \\
\hline & $(8.66)$ & $(9.56)$ & (70.98) & $(8.98)$ & (8.32) & $(3.161)$ \\
\hline
\end{tabular}

Values are presented as arithmetic mean $( \pm S D)$ concentrations $(\mathrm{pg} / \mathrm{mL})$

Compare to baseline ${ }^{\star} \mathrm{P}<0.05,{ }^{\star *} \mathrm{P}<0.01$.

\section{Figures}


A

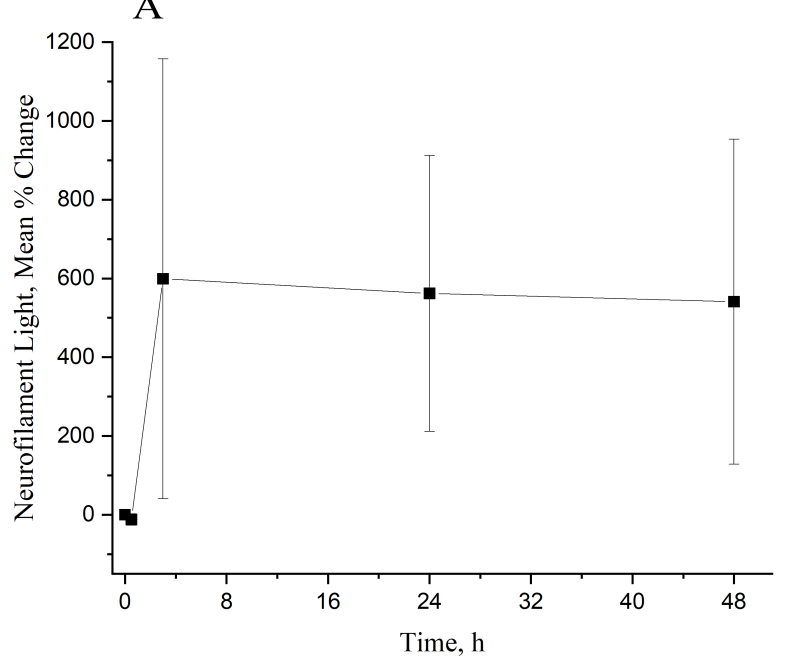

$\mathrm{C}$

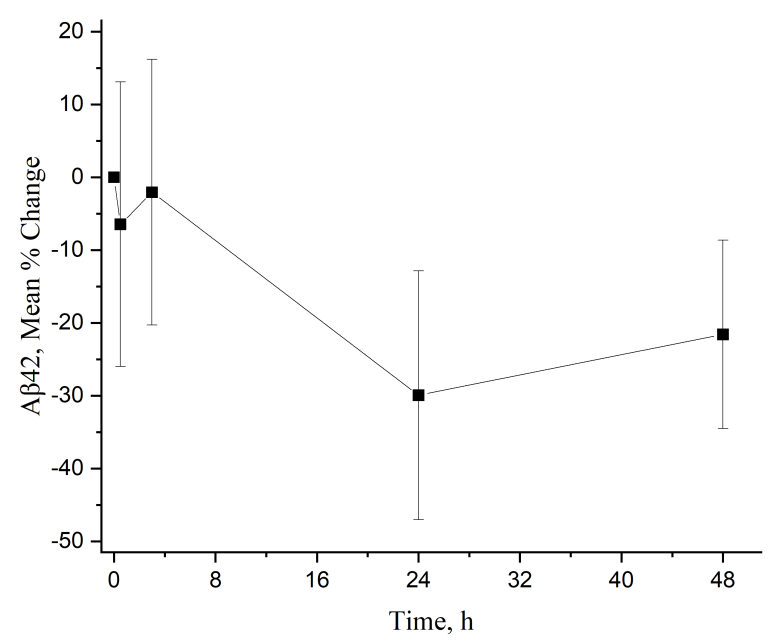

B

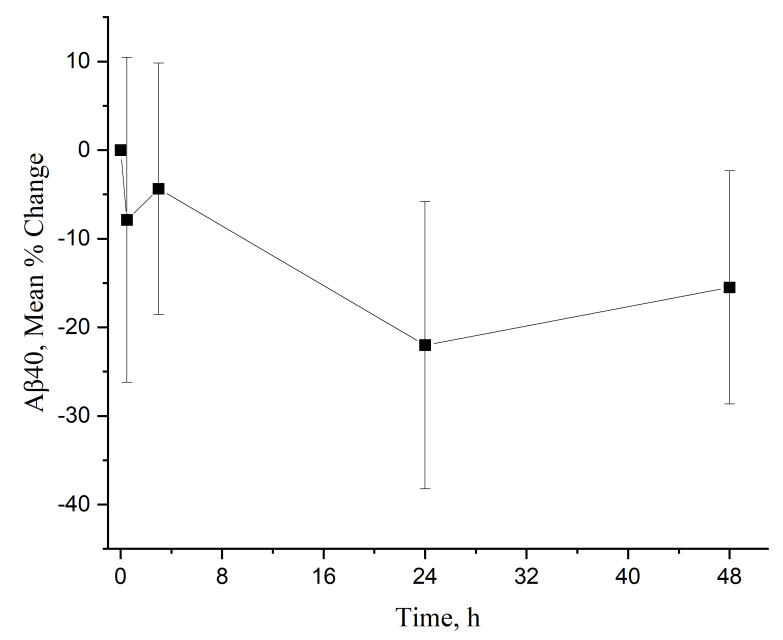

$\mathrm{D}$

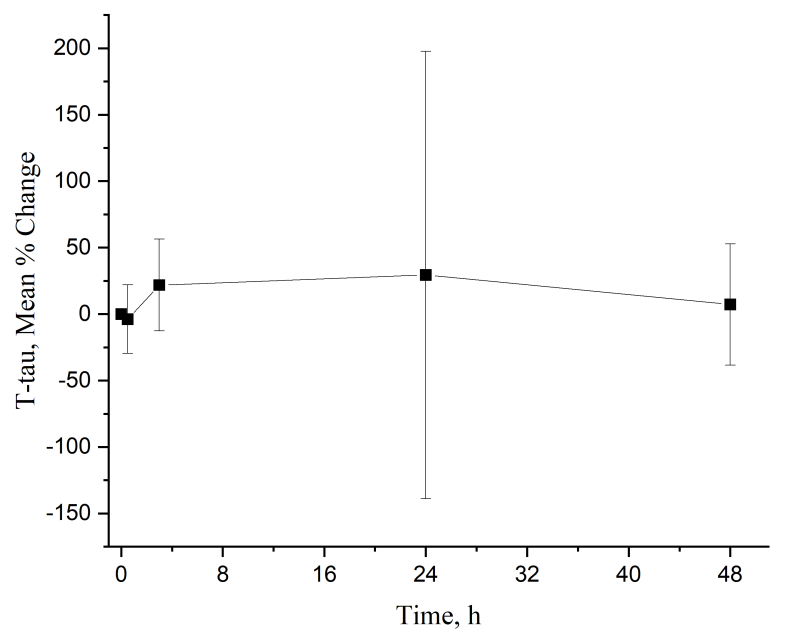

\section{Figure 1}

The changes in plasma level of neurofilament light, T-tau, $A \beta 40$ and $A \beta 42$ over time after surgery and anesthesia. The line indicates the mean, and the error bars indicate the standard deviation. 

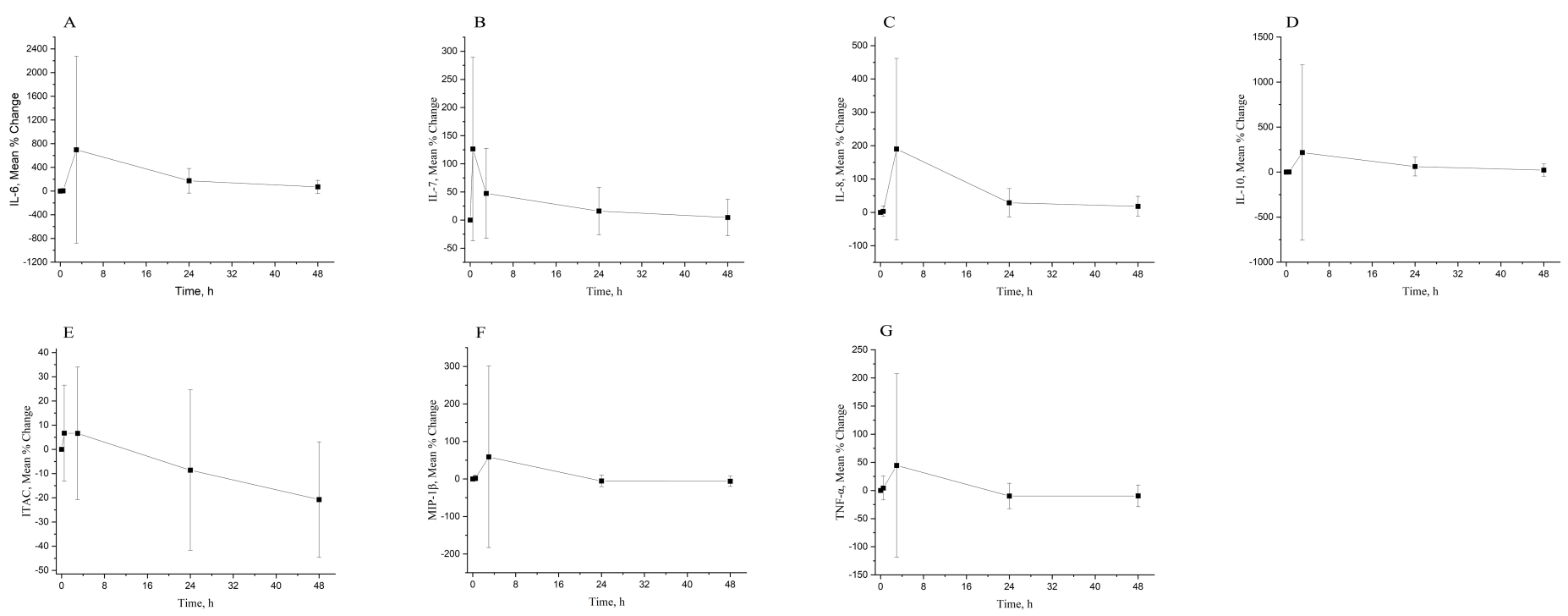

\section{Figure 2}

The changes in plasma level of inflammatory markers after surgery and anesthesia. The line indicates the mean, and the error bars indicate the standard deviation.
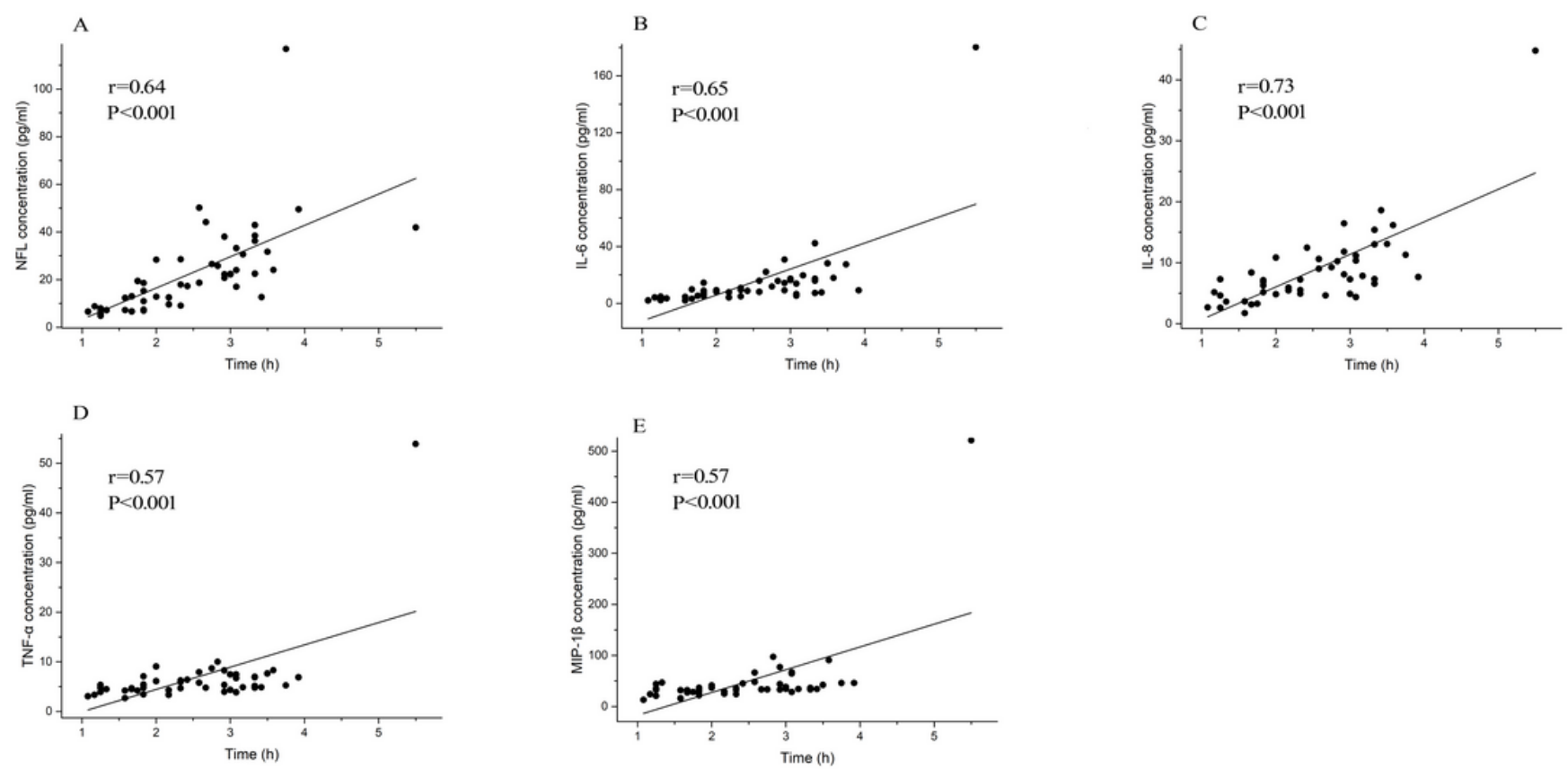

\section{Figure 3}

Correlations between the peak levels of NFL, IL-6, IL-8, TNF- $a$ and MIP-1 $\beta$ postoperatively and the duration of surgery. 

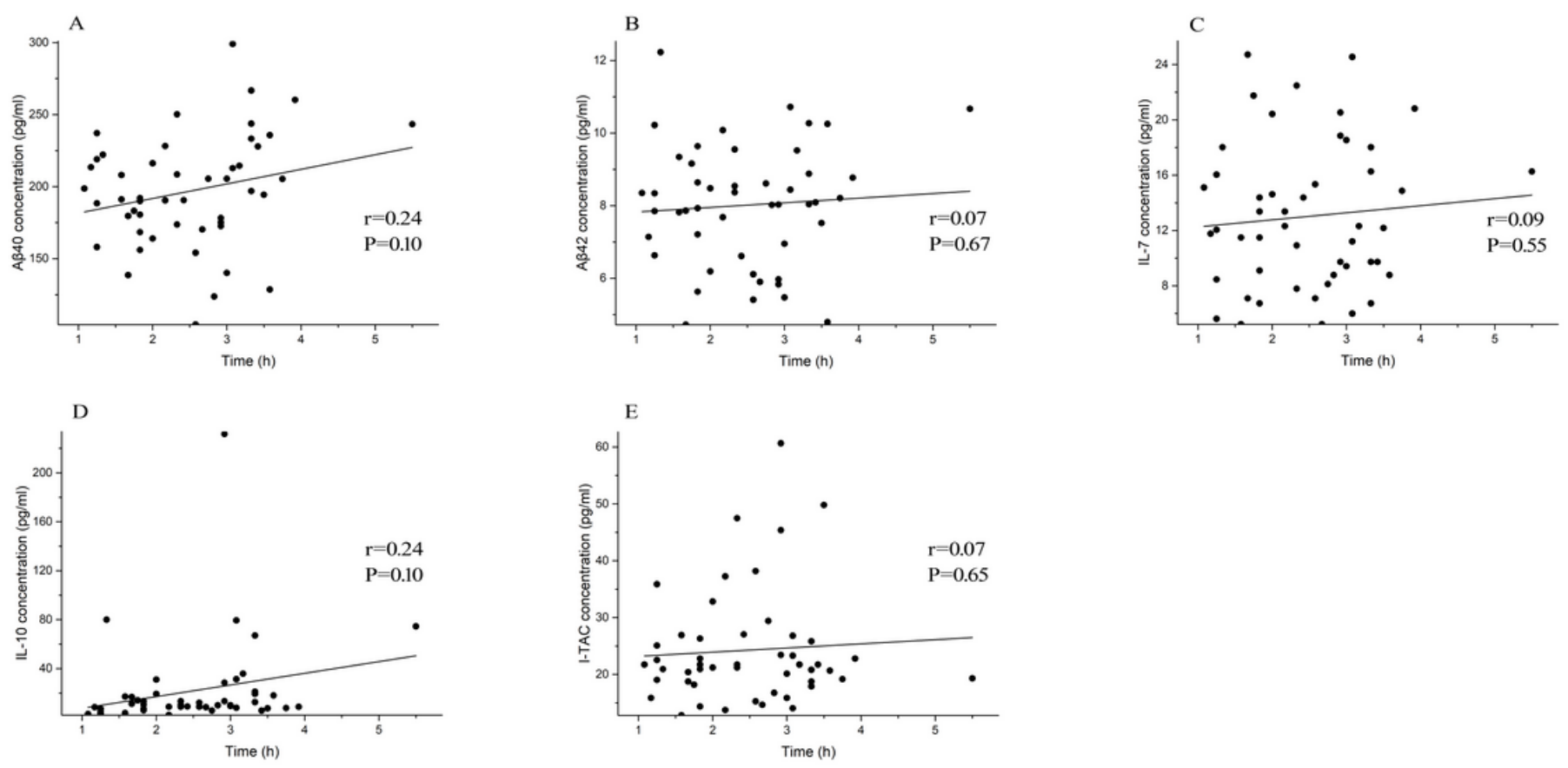

Figure 4

Correlation of peak plasma $A \beta 40, A \beta 42$, IL-7, IL-10, I-TAC and the duration of surgery.
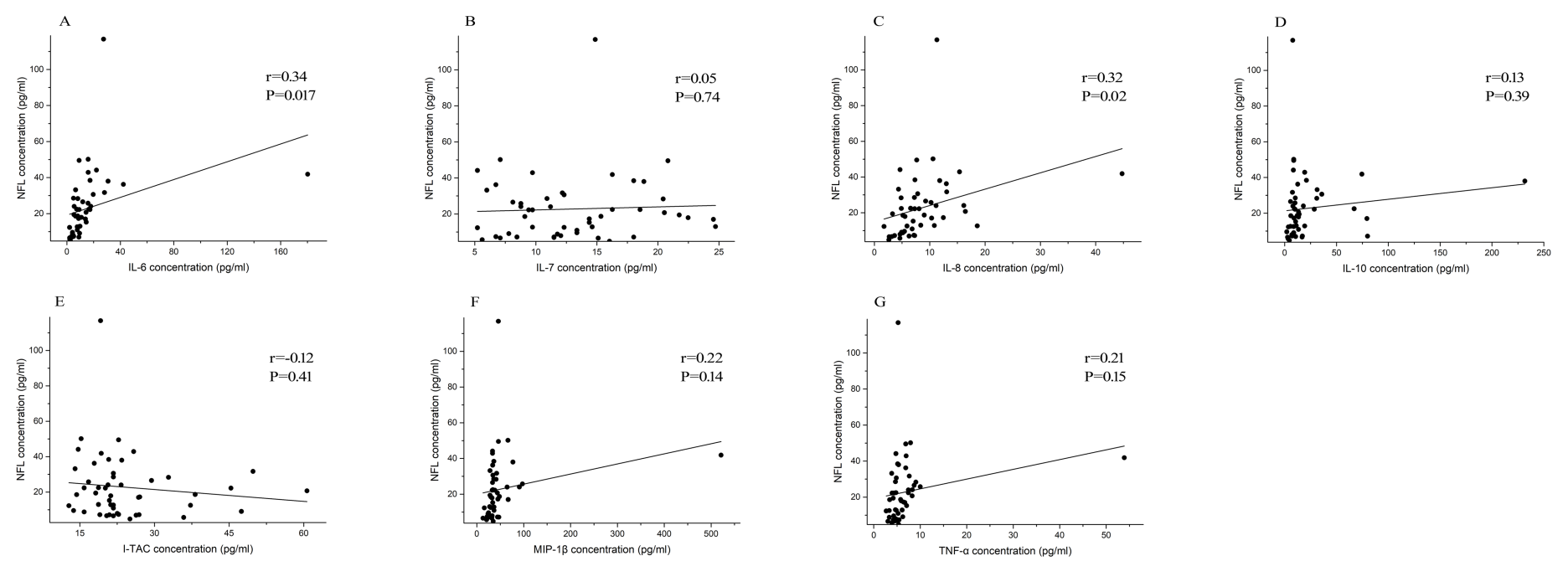

Figure 5

Correlations between the peak levels of NFL and inflammatory mediators.

\section{Supplementary Files}

This is a list of supplementary files associated with this preprint. Click to download.

- Additionalfile1S1InclusionandExclusioncriteria.docx

- Additionalfile2S2StudyflowchartFigureFigureS.tif 
- Additionalfile3plasmalevelsofcytokines.docx 